\title{
Demonstration of a Novel Positron Source Based on a Plasma Wiggler
}

\author{
D.K. Johnson*, I. Blumenfeld ${ }^{\dagger}$, C.D. Barnes ${ }^{\dagger}$, C.E. Clayton*, F.J. Decker ${ }^{\dagger}$, \\ S. Deng ${ }^{* *}$, P. Emma ${ }^{\dagger}$, M.J. Hogan ${ }^{\dagger}$, C. Huang* ${ }^{*}$ R. Ischebeck ${ }^{\dagger}$, R. Iverson ${ }^{\dagger}$, \\ C. Joshi*, T.C. Katsouleas**, N. Kirby ${ }^{\dagger}$, P. Krejcik ${ }^{\dagger}$, W. Lu*, K.A. Marsh*, \\ W.B. Mori*, P. Muggli**, C.L. O'Connell', E. Oz ${ }^{* *}$, R.H. Siemann ${ }^{\dagger}$, \\ D. Walz ${ }^{\dagger}$ and M. Zhou* \\ *University of California, Los Angeles, CA 90095, USA \\ ${ }^{\dagger}$ Stanford Linear Accelerator Center, Stanford, CA 94309, USA \\ ** University of Southern California, Los Angeles, CA 90089, USA
}

\begin{abstract}
A new method for generating positrons has been proposed that uses betatron X-rays emitted by an electron beam in a high-K plasma wiggler. The plasma wiggler is an ion column produced by the head of the beam when the peak beam density exceeds the plasma density. The radial electric field of the beam blows out the plasma electrons transversely, creating an ion column. The focusing electric field of the ion column causes the beam electrons to execute betatron oscillations about the ion column axis. If the beam energy and the plasma density are high enough, these oscillations lead to synchrotron radiation in the $1-50 \mathrm{MeV}$ range. A significant amount of electron energy can be lost to these radiated $\mathrm{X}$-ray photons. These photons strike a thin $\left(.5 X_{o}\right)$, high$\mathrm{Z}$ target and create $e^{+} / e^{-}$pairs. The experiment was performed at the Stanford Linear Accelerator Center (SLAC) where a $28.5 \mathrm{GeV}$ electron beam with $\sigma_{r} \approx 10 \mu \mathrm{m}$ and $\sigma_{z} \approx 25 \mu \mathrm{m}$ was propagated through a neutral Lithium vapor $(\mathrm{Li})$. The radial electric field of the dense beam was large enough to field ionize the $\mathrm{Li}$ vapor to form a plasma. The positron yield was measured as a function of plasma density, ion column length and electron beam pulse length. A computational model was written to match the experimental data with theory. The measured positron spectra are in excellent agreement with those expected from the calculated X-ray spectral yield from the plasma wiggler. After matching the model with the experimental results, it was used to design a more efficient positron source, giving positron yields of $0.44 e^{+} / e^{-}$, a number that is close to the target goal of $1-2 e^{+} / e^{-}$for future positron sources.
\end{abstract}

Keywords: Positron Production, Beam-Plasma Interactions, Plasma Accelerators, X-ray Beams PACS: 52.29.Ye, 52.40.MJ, 52.25 Jm, $41.75 \mathrm{Ht}$

\section{INTRODUCTION}

High energy physicists use electron-positron collisions to validate the predictions of various field theories. The positrons $\left(e^{+}\right)$needed for the collisions are currently produced by bombarding a high-Z, solid target that is several radiation lengths thick with a high energy electron beam [1]. The resulting interaction creates bremsstrahlung photons which can interact with the atomic nuclei of the target producing electron-positron pairs. Positron beam requirements for future linear colliders suggest that this type of "thicktarget", bremsstrahlung $e^{+}$source may fail from thermal stress since multiple scattering of the $e^{-}$beam deposits a large amount of energy into the target.

There have been several alternative designs for next generation positron sources. All

\author{
CP877, Advanced Accelerator Concepts: $12^{\text {th }}$ Workshop, \\ edited by M. Conde and C. Eyberger \\ (C) 2006 American Institute of Physics 978-0-7354-0378-9/06/\$23.00
}




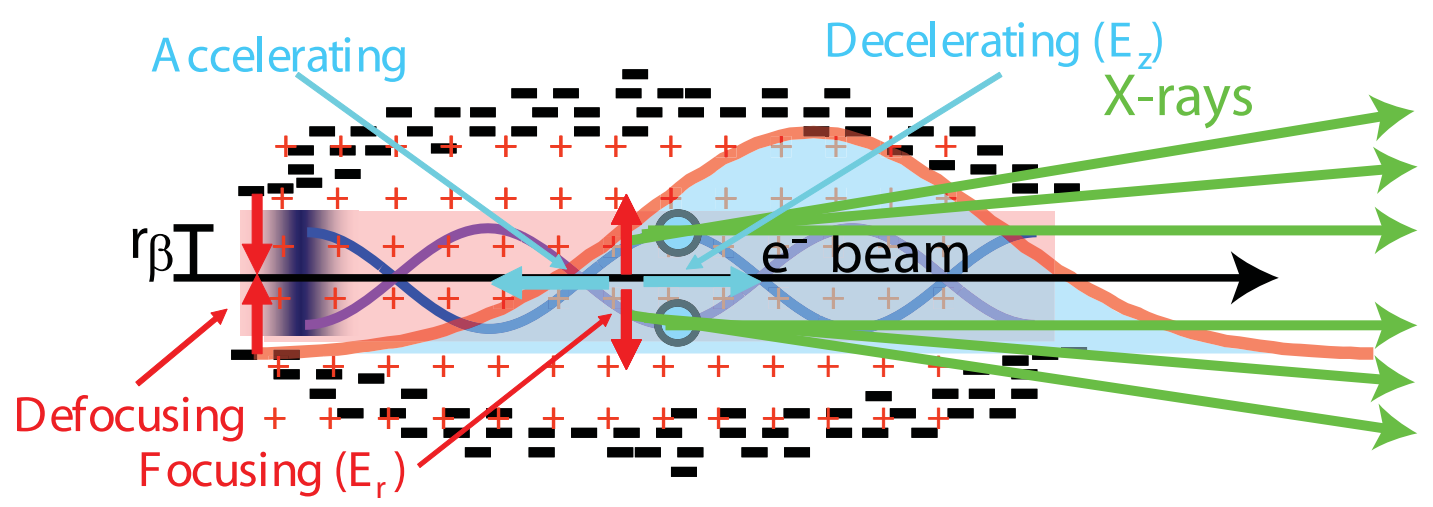

FIGURE 1. Schematic of electron betatron motion within a plasma ion column. An example of an individual electron radius is denoted by $r_{\beta}$. For a beam of electrons, there will be a distribution of radii, and each radial bin of electrons will radiate distinct $\mathrm{X}$-ray spectra.

of the methods considered produce positrons after colliding MeV X-rays with a thin, high-Z target. Here, "thick" and "thin" is relative to a radiation length. The source must also have a high conversion efficiency of drive beam energy to $\mathrm{MeV} \mathrm{X}$-ray photons since an average photon energy of $10-30 \mathrm{MeV}$ is desired for efficient production of positrons [2]. Photo-production of positrons is seen as the solution for future linear colliders, but the methods for efficiently producing the X-rays are being studied. One proposal uses a helical undulator to produce polarized photons in the 5-10 MeV range [3]. Another proposed source uses Compton back-scattering of an electron beam off a circularly polarized laser beam to produce polarized photons in this same energy range [4]. Both of these schemes are attractive because they can generate polarized positrons that are highly desirable for high energy particle physics. It has also been suggested that a Tungsten (W) crystal could be employed as an atomic undulator to produce $\mathrm{MeV}$ $\mathrm{X}$-rays. In this scheme, the beam undulates within the crystal creating $\mathrm{MeV}$ photons near the entrance region and creates pairs towards the exit region of the crystal [5]. It is imperative that any new source overcomes the thermal stress and shock-wave issues associated with the aforementioned Bremsstrahlung source. We propose an alternative method of generating X-ray photons in the spectral range of interest to produce positrons and present results from an experiment conducted at the Stanford Linear Accelerator Center (SLAC) where a $28.5 \mathrm{GeV}$ electron beam was used in a proof-of-principle demonstration of this scheme. Our scheme utilizes a plasma wiggler to wiggle the electrons via betatron oscillations. The plasma wiggler is an ion column produced by the beam itself. The radial electric field of the ion column causes an oscillating motion of the off-axis electrons (called betatron oscillations) as they propagate through the ion column. This wiggling motion creates a large broadband flux of $\mathrm{MeV} \mathrm{X}$-rays. These $\mathrm{X}$-rays were collided with a thin, high-Z target to produce positrons.

The experimental work presented in this paper was performed in the underdense regime. This means that the electron beam density $n_{b}$ was greater than the plasma density $n_{p e}$. Figure 1 shows how an electron beam produces an ion column as it propagates through a uniform, underdense plasma. In such a case, the head of the electron beam 
expels all the plasma electrons radially, leaving behind an ion column. The ion column exerts a restoring force on the blown out plasma electrons which rush back toward the axis, overshoot and set up a wake oscillation. In the experiment, the plasma wavelength $\lambda_{p}$ was about four times greater than the longitudinal bunch length of the electron beam $\sigma_{z}$. The longitudinal wakefield decelerates most of the beam electrons. However, it is the transverse motion of the electrons that is of interest here. The off-axis electrons are attracted by the radial electric field of the ion column and oscillate about the beam axis at the betatron frequency $\omega_{\beta}=\omega_{p} / \sqrt{2 \gamma_{b}}$ where $\omega_{p}$ is the plasma frequency and $\gamma_{b}$ is the electron Lorentz factor. As the beam electrons undergo these betatron oscillations, they emit X-ray radiation in a narrow cone in the forward direction which is used for pair production.

In previous work, betatron motion of electrons in an ion column with a density $n_{p e} \approx$ $10^{14} \mathrm{~cm}^{-3}$ was seen to generate $\sim 6-10 \mathrm{keV} \mathrm{X}$-rays in the direction of propagation of the electron beam [6]. Because of the low plasma density in that experiment, the effective wiggler strength was on the order of 1 , comparable to modern magnetic undulator systems. However, for positron production with energies of interest for accelerators, one needs X-rays in the 1-50 MeV energy range. Recall that the minimum photon energy needed for the production of $e^{+}-e^{-}$pairs is $1.022 \mathrm{MeV}$. To produce such high energy $\mathrm{X}$-rays, the work here utilizes much higher plasma densities $\left(\sim 10^{17} \mathrm{~cm}^{-3}\right)$, and effective wiggler strengths up to 500. It is not practical to produce long uniform columns of such high density plasma using the UV photo-ionization technique of previous experiments. Therefore, the electric field of the electron beam was used to ionize the neutral gas. In order to exceed the field ionization threshold, the beam density must be high which in turn means that the beam pulse length had to be on the order of $25 \mu \mathrm{m}$ with a beam radius of roughly $10 \mu \mathrm{m}$. Such short, dense electron beam pulses were available at the Final Focus Test Beam (FFTB) at the Stanford Linear Accelerator Center (SLAC).

The plasma wiggler in this scheme is an ion column that exerts a focusing force on the beam leading to electron betatron oscillations [7]. The radial electrostatic field of the ion column which produces these oscillations is given by $E_{r}(r)=\left(n_{p e} e / 2 \varepsilon_{o}\right) r$ (SI units) where $n_{p e}$ is the plasma density and $e$ is the electron charge $[6,8]$.

The fundamental parameter describing the plasma wiggler is the wiggler strength defined as $K=\gamma k_{\beta} r_{o}$ where $k_{\beta}=\omega_{p} / c \sqrt{2 \gamma}$ is the betatron wavenumber, $\omega_{p}$ is the plasma frequency, $\gamma$ is the beam Lorentz factor and $r_{o}$ is the maximum radius of the oscillating electron. Note that the electrons in a beam will have a distribution about $r_{o}$. The spectrum of the betatron radiation has frequencies

$$
\omega_{n}=\frac{n 2 \omega_{\beta} \gamma^{2}}{1+K^{2} / 2+(\gamma \theta)^{2}}
$$

where $\theta$ is the angle between the axial motion of the beam and the observation point and $n$ is the harmonic number [6,8]. When $K \gg 1$, higher harmonic radiation dominates the spectrum, and since $K$ is linearly proportional to $r_{o}$, individual electrons at different radii have different radiated frequencies resulting in a broadband spectrum. This spectrum is characterized by a critical frequency $\omega_{c}$ and a critical harmonic number $n_{c}$. For on-axis radiation $(\theta=0)$, these quantities are $\omega_{c}=3 K \gamma^{2} \omega_{\beta} / 2$ and $n_{c}=3 K^{3} / 8[6,8]$. 
The electron energy loss is given by the relativistic Larmor formula. Using the betatron orbits due to the radial electrostatic potential, the energy loss per unit length is

$$
\frac{d W_{\text {loss }}}{d z}=\frac{1}{3} r_{e} m_{e} \gamma^{2} \omega_{\beta}^{2} K^{2} \propto\left(\gamma^{2}, n_{p e}^{2}, r_{o}^{2}\right)
$$

where $m_{e}$ is the electron mass and $r_{e}$ is the classical electron radius [6, 8]. As an example, in an ion column of density $n_{i}=3 \times 10^{17} \mathrm{~cm}^{-3}$, an electron with an energy of $28.5 \mathrm{GeV}$ and an $r_{o}$ of $10 \mu \mathrm{m}$ experiences a wiggler strength $K$ of 173 with an on-axis critical photon energy of roughly $50 \mathrm{MeV}$, ideal for positron production. Equation 2 shows that this electron radiates $\sim 4.3 \mathrm{GeV} / \mathrm{m}$. Thus, such a high-K plasma wiggler is well-suited for converting the electron beam energy into a large number of multi-MeV photons, a necessary condition for a practical positron source.

\section{SPECTRUM CALCULATION}

The radiation spectrum from the oscillating electrons in a plasma wiggler was computed using the formalism described in Ref. [9], which is well-suited for a high-K wiggler. It is well-known that synchrotron radiation is emitted in a cone angle $\theta=1 / \gamma$ around the instantaneous momentum vector $\vec{p}$ of the particle. Assuming betatron motion in the $\mathrm{y}-\mathrm{z}$ plane, we get a characteristic divergence angle of $\theta_{x}=1 / \gamma$ perpendicular to the particle plane and $\theta_{y}=K / \gamma$ in the particle oscillation plane. The $\theta_{y}$ dependence arises since $\vec{p}$ is constantly changing its transverse component throughout the betatron orbit. When $K \gg 1$, only certain phases along the betatron trajectory contribute to the observed spectrum in the far-field as shown in figure 2. These phases correspond to the maximum positive and negative displacement regions of the electron trajectory where the acceleration is the greatest and, locally, $\vec{p}$ is parallel to the vector defining the observation point in the far-field. This allows for a "synchrotron-like" spectrum approximation for the photons by Taylor-expanding the electron orbit around these contributing phases [saddle-point method] [9].

The far-field spectrum of the photon beam produced by a plasma wiggler has been computed using the saddle-point method. The result gives the energy per unit frequency per unit solid angle $\left(\frac{d^{2} W}{d \omega d \Omega}\right)$ as shown in figure 3. A 3-D and 2-D contour plot of the radiated energy per unit solid angle $\left(\frac{d W}{d \Omega}\right)$ is shown in figure $4 \mathrm{a}$ and $4 \mathrm{~b}$, respectively. Note that $\theta=1 / \gamma$ perpendicular the to electron oscillation plane, and $\theta=K / \gamma$ parallel to the electron oscillation plane. The result $\left(\frac{d^{2} W}{d \omega d \Omega}\right)$ is integrated with respect to $d \omega$ and $d \Omega$, and the agreement with theory is excellent as seen in table 1 . The X-ray spectrum is computed for an angle of $0.1 \mathrm{mrad}$ corresponding to the $8 \mathrm{~mm}$ photon beam in the experiment. The X-ray spectrum as a function of position is then input into the ElectronGamma Shower 4 code (EGS4) [10]. Within EGS4, the photons collide with the W target generating the positrons. The positrons emanating from the rear of the target are propagated through the appropriate magnetic transport matrices to the location of the surface barrier detectors (SBDs). 


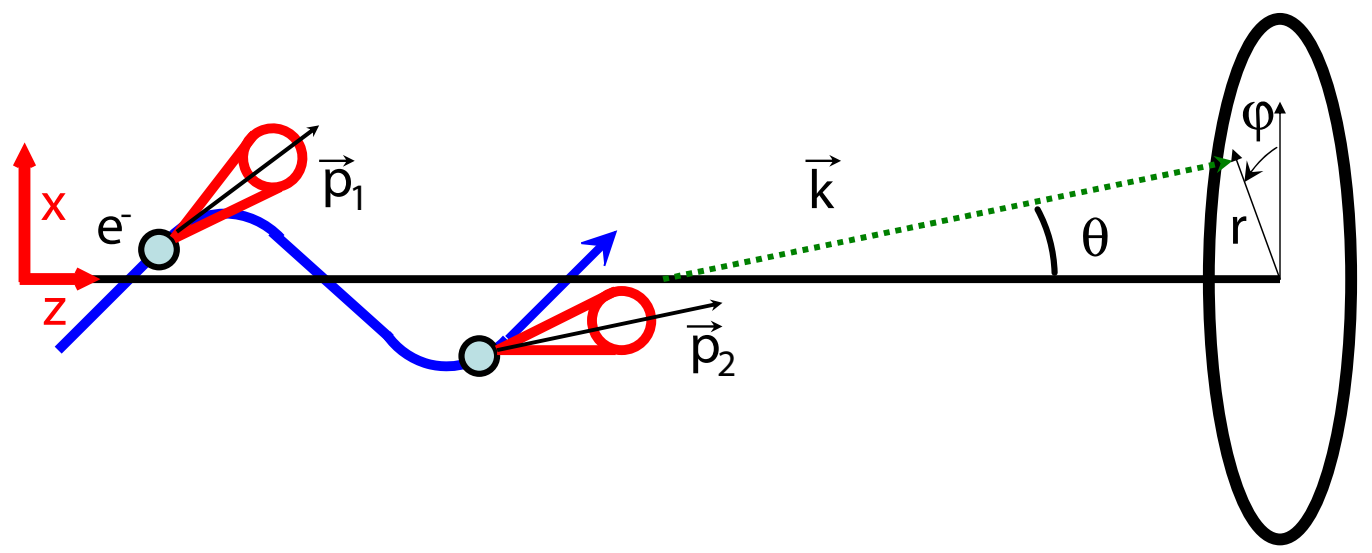

FIGURE 2. Schematic of the Saddle-Point Method. $\vec{p}_{1}$ will not contribute to the spectrum at the farfield position determined by $\vec{k}$ since $\vec{k} \cdot \vec{p}_{1}$ is small. However, $\vec{p}_{2}$ will have a substantial contribution to the far-field at that point since $\vec{k} \cdot \vec{p}_{2}$ is large.

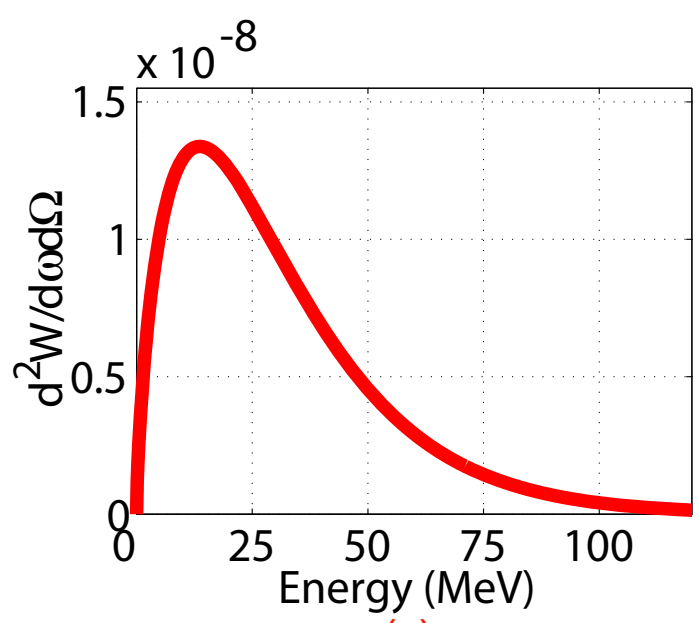

(a)

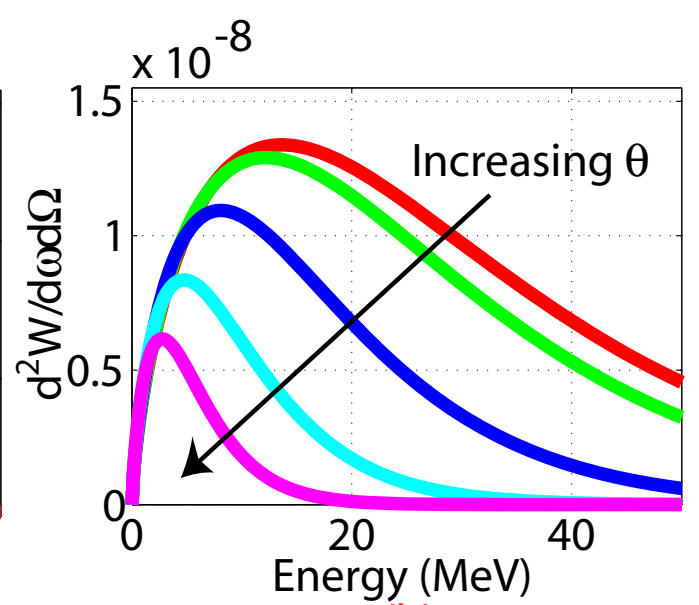

(b)

FIGURE 3. Solutions to the Saddle-Point method. This assumes an electron with $E=28.5 \mathrm{GeV}$, $r=10 \mu m$ and $n_{p e}=1 \times 10^{17} \mathrm{~cm}^{-3}$. The units of $d^{2} W / d \omega d \Omega$ are $e V \cdot s / \Omega$. (a) At $\phi=0$ and $\theta=0$. (b) At $\phi=90$ with increasing $\theta$.

TABLE 1. The comparison of the Larmor formula [8] and computed values for the total energy loss from one electron undergoing four betatron oscillations $\left(N_{\beta}=4\right)$.

\begin{tabular}{c|c|c|c}
$n_{p e}\left(1 / \mathrm{cm}^{3}\right)$ & Larmor $(\mathrm{MeV})$ & Calculated $(\mathrm{MeV})$ & Error \\
\hline $1 \times 10^{17}$ & 66.5 & 66.0 & .00752 \\
\hline $2 \times 10^{17}$ & 188.1 & 187.8 & .00159 \\
\hline $3 \times 10^{17}$ & 346 & 348 & .00578 \\
\hline
\end{tabular}




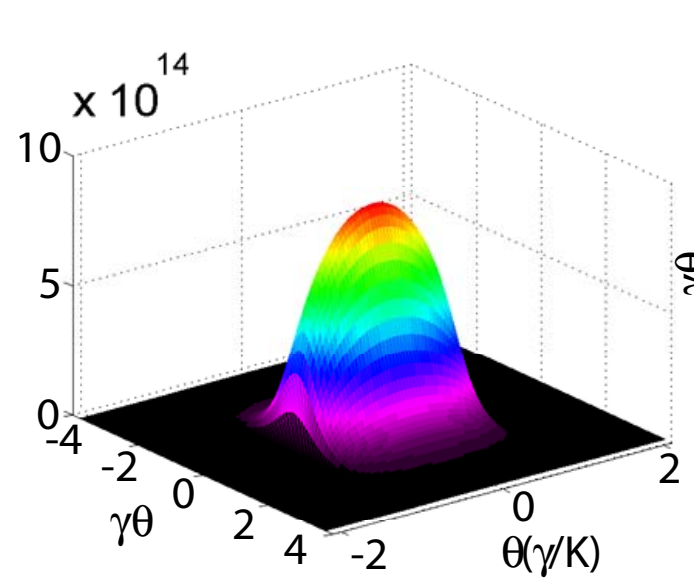

(a)

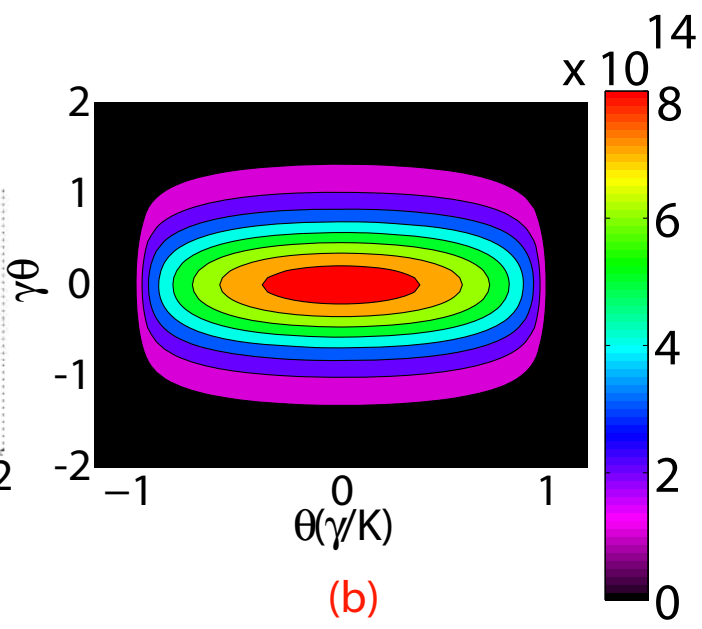

(b)

FIGURE 4. Contour plots of the total radiated energy in the far-field using the Saddle-Point method. This assumes an electron with $E=28.5 \mathrm{GeV}, r=10 \mu \mathrm{m}$ and $n_{p e}=1 \times 10^{17} \mathrm{~cm}^{-3}$. The units of $d \mathrm{~W} / d \Omega$ are $\mathrm{eV} / \Omega$; (a) 3-D. (b) 2-D projection of (a).

\section{EXPERIMENTAL SETUP}

The experiment [11] was carried out in the FFTB at SLAC. A schematic of the experimental setup is shown in Fig. 5. A $28.5 \mathrm{GeV}$ beam containing approximately $N_{b o}=1.7 \times 10^{10}$ electrons was focused into a lithium (Li) vapor of variable length of $10-30 \mathrm{~cm}$ and variable neutral density of $n_{o}=1-30 \times 10^{16} \mathrm{~cm}^{-3}$. After propagating through a beryllium window which separates the plasma source from the FFTB beamline vacuum, the beam has emittances $\varepsilon_{x} \approx 5 \varepsilon_{y} \approx 2.0 \times 10^{-9} \mathrm{~m}-\mathrm{rad}$. For our nominal waist location, the beam is round with $\sigma_{x, y} \approx 10 \mu \mathrm{m}$ resulting in $\beta_{x} \ll \beta_{y}$. If the beam spot size at the plasma entrance is small enough or the current density is high enough, the transverse electrostatic field completely field-ionizes the Li vapor [12, 13], creating a singly-ionized plasma in the risetime of the electron beam. If the beam density $n_{b}$ is greater than the plasma density $n_{p e}$, the space charge force of the beam rapidly expels the plasma electrons and creates a pure ion column $\left(n_{p e}=n_{i}\right)$. The upper bound on $n_{p e}$ is limited by this constraint. The focusing force $E_{r}$ of this ion column causes the beam electrons to oscillate about the column axis and radiate betatron $\mathrm{X}$-rays [6]. It also exerts a restoring force on the expelled plasma electrons which rush back, overshoot the ion column axis, and set up a wake oscillation. It addition to radiating some of their energy due to betatron oscillations, the electrons can lose energy because of the retarding effect of the wakefield [14]. In the example given earlier, an electron can lose up to $40 \mathrm{GeV} / \mathrm{m}$ to the wakefield almost an order of magnitude greater than that to X-ray radiation.

The transverse beam profile was recorded both upstream and downstream of the plasma by imaging the optical transition radiation (OTR) produced by the beam traversing two $1 \mu \mathrm{m}$ thick titanium foils. With the $\mathrm{Li}$ vapor removed, these profiles allow for the determination of the vacuum beam waist location. Coherent transition radiation (CTR) in the THz range was detected after the bunch propagated through another $1 \mu \mathrm{m}$ thick Ti foil upstream of the plasma. The CTR energy was measured using a pyroelectric 

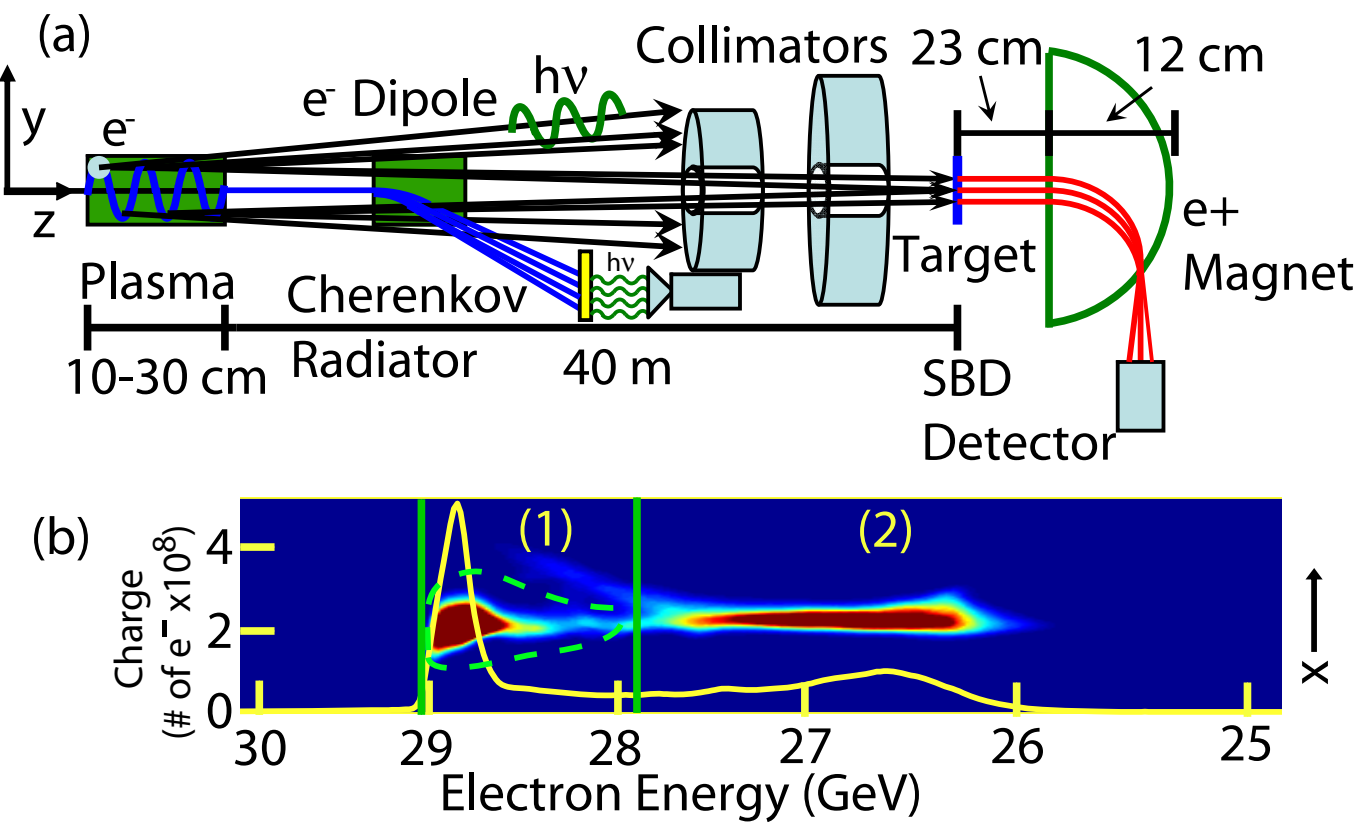

FIGURE 5. (a) An Experimental Schematic. (b) A typical electron energy spectrum image using a Cherenkov radiator diagnostic and a lineout after summing the spectrum over $\mathrm{x}$ (yellow solid line). In the absence of a plasma, the electron beam spectrum (dotted contour) is in region (1). When the beam traverses the plasma, some of the charge is transferred from region (1) to region (2) as the $e^{-}$lose energy.

detector. For a fixed $N_{b o}$, it is correlated to the inverse pulse length $\left(1 / \sigma_{z}\right)$ of the electron beam $[11,15]$, and ultimately to the wake amplitude driven by the electron beam propagating through the plasma (see later). A CTR energy of 400 (AU) corresponds to $\sigma_{z}$ of the beam of $\sim 20 \mu m$.

The radiated betatron X-rays propagate in vacuum $40 m$ downstream to the positron convertor target. This distance is necessary due to the beamline layout of the FFTB. To minimize potential background signals on the positron detectors, the maximum photon angular divergence of $\theta_{\max }=K_{\max } / \gamma \approx 9 \mathrm{mrad}$ was collimated down to $0.1 \mathrm{mrad}$ using two $10 \mathrm{~cm}\left(25 X_{o}\right)$ long tungsten $(\mathrm{W})$ collimators with diameters of $6.4 \mathrm{~mm}$ and $12.7 \mathrm{~mm}$, located $25 \mathrm{~m}$ and $30 \mathrm{~m}$ from the plasma, respectively. These created an $8 \mathrm{~mm}$ collimated photon beam at the positron target. The X-rays interacted with a $1.7 \mathrm{~mm}(\approx .5$ radiation lengths) thick $\mathrm{W}$ target producing electron-positron pairs. The resulting positrons, up to an energy of $20 \mathrm{MeV}$ were imaged in a magnetic spectrometer with an aperture of roughly $12 \mathrm{~mm}$ (vertical) by $32 \mathrm{~mm}$ (horizontal) and detected using $1 \mathrm{~mm}$ thick silicon surface barrier detectors (SBDs) with a surface area of $49 \mathrm{~mm}^{2}$.

The electron beam exiting the plasma was steered by a dipole magnet to separate it from the X-rays. It was subsequently imaged on a Cherenkov radiator to record the spectrum of the beam electrons. An example of one such spectrum with and without the plasma is shown in Fig. 5(b) for $n_{p e}=1 \times 10^{17} \mathrm{~cm}^{-3}$ and a plasma length $L_{p}=11 \mathrm{~cm}$. These images were used to estimate the actual number of electrons that interact with the ion column. 


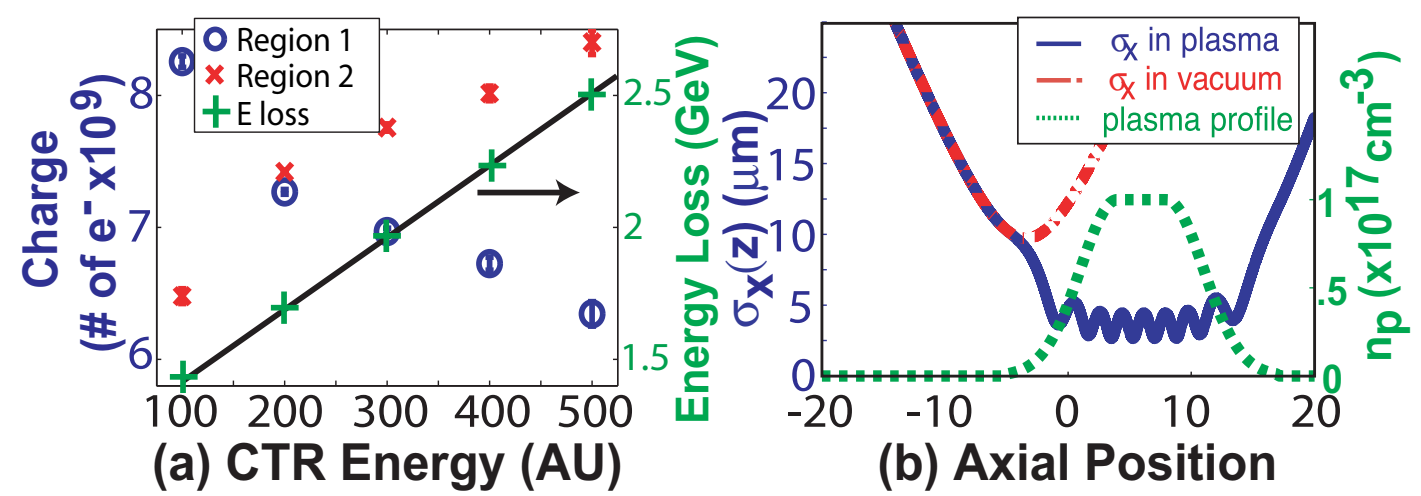

FIGURE 6. (a) The charge $\left(N_{b}\right)$ in region (1) of the bunch (red $\mathrm{x}$ ) and in region (2), the radiating portion of the bunch (blue circle) versus the CTR signal $\left(\sim 1 / \sigma_{z}\right)$. At a CTR of 100, there has already been charge transfer from region (1) to region (2). Also plotted is the average beam energy loss for the radiating electrons within the $11 \mathrm{~cm}$ plasma (wake+radiation) with $n_{p e}=1 \times 10^{17} \mathrm{~cm}^{-3}$. (b) The calculated variation of $\sigma_{x}$ inside the plasma using a beam envelope model (see text for details). The green dotted curve is the plasma density profile.

\section{RESULTS}

The three critical parameters which determine the positron yield are the number of electrons $N_{b i}$ that participate in betatron oscillations, the effective energy of the beam electrons $\gamma_{b i}$ and the spot-size of the beam in the plasma $\sigma_{i: x, y}$. Here, we experimentally estimate $N_{b i}$ and $\gamma_{b i}$ as described below, and calculate the latter based on the excellent agreement between the spot size observed on the OTR screen as the beam exits the plasma and that predicted by a beam envelope model $[7,16]$.

We estimate $N_{b i}$ the following way. As the beam enters the Li vapor, it begins to form a plasma once the threshold for field-ionization is exceeded. The beam electrons do work in pushing out the plasma electrons and thus lose energy. In Fig. 5(b), one can see that there is a transfer of charge from region (1) (no plasma condition) to region (2) when the beam produces ionization of $\mathrm{Li}$. For a given density of $\mathrm{Li}$, the smaller the $\sigma_{z}$ of the beam, the earlier the ionization occurs and a larger fraction of the beam electrons lose energy. This is shown in Fig. 6(a), for $n_{p e}=1 \times 10^{17} \mathrm{~cm}^{-3}$, where one can clearly see this transfer of charge from region (1) to region (2) due to energy loss as the CTR energy increases. For instance, at a CTR energy of 200 (AU), $7.2 \times 10^{9}$ electrons lose energy and therefore must reside in the ion column. Figure 6(a) also shows the mean energy lost by the charge as it is transferred from region (1) to region (2) of Fig. 5(b) as a function of CTR energy. We assume that this loss scales linearly with distance in the plasma and adjust the beam energy $\gamma_{b i}$ at each saddle-point accordingly.

The radiated x-ray energy, and therefore the positron yield, was first optimized by adjusting the position of the beam waist with respect to the plasma entrance. This changes the size of the focused spot size within the plasma column which in turn controls the positron yield. For instance, the optimum beam waist location was found to be at an axial position of $\sim-5 \mathrm{~cm}$ (at $\sim 10 \%$ of the peak Li density) as shown in Fig. 6(b) with $n_{p e}=1 \times 10^{17} \mathrm{~cm}^{-3}$. As the beam enters the neutral Li gas whose profile is shown by the 
TABLE 2. This table lists the pertinent simulation parameters for each of the cases in figure $7 \mathrm{a}$.

\begin{tabular}{c|c|c|c} 
Case & 1 & 2 & 3 \\
\hline Density $\left(\mathrm{cm}^{-3}\right)$ & $1 \times 10^{17}$ & $6.4 \times 10^{16}$ & $3.3 \times 10^{16}$ \\
\hline Oven FWHM $(\mathrm{cm})$ & 11 & 14 & 16 \\
\hline Saddle-Points & 6 & 6 & 5 \\
\hline Average CTR signal $\left(1 / \sigma_{z}\right)$ & $\sim 85$ & $\sim 175$ & $\sim 300$ \\
\hline Ion Column Charge $\left(N_{b i}\right)$ & $7.2 \times 10^{9}$ & $8 \times 10^{9}$ & $8.25 \times 10^{9}$ \\
\hline Ion Column Radius $\left(\sigma_{i: x, y}[\mu \mathrm{m}]\right)$ & 4 & 5 & 8 \\
\hline Wakeloss $\left(\gamma_{b}[\mathrm{GeV} / \mathrm{m}]\right)$ & 25 & 15 & 11 \\
\hline
\end{tabular}

green line in Fig. 6(b), the Li is ionized creating a low density plasma. This plasma acts as a thick lens that rapidly focuses the beam to a spot size smaller than its vacuum value before it begins betatron oscillations within the high density body of the plasma column [16]. Once in this region, the beam envelope oscillates with a maximum $\sigma_{i: x, y} \simeq 4 \mu \mathrm{m}$ where the electrons radiate most of the synchrotron energy. Using the 3-D particle-in-cell code QuickPIC [17], which is a fully nonlinear, three-dimensional particle-in-cell code that utilizes the quasi-static approximation and includes field ionization and radiation loss, we have confirmed that the envelope model gives a very good estimate of the beam spot size inside the plasma [18].

The values of $N_{b i}, \gamma_{b i}$ and $\sigma_{i: x, y}$ used in the calculation are obtained for each density as described above. The resulting parameters are shown in table 2. Figure 7(a) plots the comparison between the experimentally measured positron spectrum and the calculated spectrum for three different plasma densities. The absolute agreement between the two is excellent, giving confidence to our ability to calculate yields for other parameters. By changing the polarity of the magnet, the electron spectrum was also measured. The absolute yield and spectral shape were similar to that of the positrons, as expected from $e^{+}-e^{-}$pair production in the convertor target.

According to Eqn (2), if $n_{p e}$ is changed while keeping all other variables constant $\left(N_{b}, r_{o}, \gamma_{b}\right)$, the radiated energy and therefore the positron yield should scale as $n_{p e}^{2}$ as long as the wake losses are negligible. Figure 7(b) shows the measured positron yield versus density in the range of $n_{p e}=1-10 \times 10^{16} \mathrm{~cm}^{-3}$. The positron yield increases, but not with the expected $n_{p e}^{2}$ scaling. First, as mentioned earlier, the edges of the plasma create a lens which in turn leads to a smaller spot size at higher plasma densities, and therefore a reduced X-ray radiation yield. Second, the maximum ion column radius scales as $n_{p e}^{-1 / 2}$ for a Gaussian beam [19]. Therefore, at the highest $n_{p e}$, there are fewer beam electrons residing in the ion column. Third, as the density increases, the beam begins to deposit larger amounts of energy into the plasma wake, further depressing the positron yield. When these three effects are folded into the calculation, we find that the total calculated yield is in very good agreement with the measured yield as shown in Fig. 7(b).

Figure 8(a) shows how the total yield in the energy range 4-20 MeV varies as a function of the CTR energy for $n_{p e}=1 \times 10^{17} \mathrm{~cm}^{-3}$ and an $11 \mathrm{~cm}$ plasma. At a given 


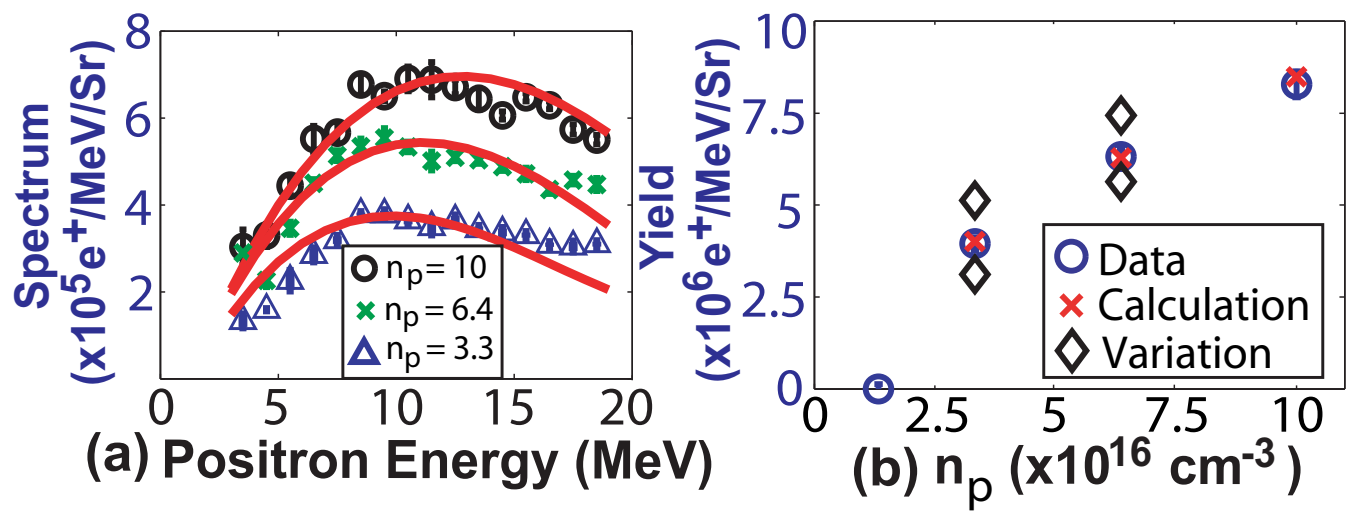

FIGURE 7. (a) Measured and calculated positron spectra for three $n_{p e}$ values. All values are $\times 10^{16} \mathrm{~cm}^{-3}$. (b) Integrated positron yield in the $4-20 \mathrm{MeV}$ energy range versus the plasma density. The black diamonds show the variation in yield with $\pm 0.5 \mu \mathrm{m}$ changes to $\sigma_{x, y}$.

density, as the CTR energy increases, the mean energy loss also increases reaching nearly $2.6 \mathrm{GeV}$ at a CTR energy of 500 as seen in Fig. 6(a). Since the betatron photon emission scales as $\gamma_{b}^{2}$ one might expect the total positron yield to go down as the CTR energy increases. Experimentally, the total yield first increases mainly because number of particles that radiate in the ion column increases up to a CTR energy of 300 as shown in Fig. 6(a). However, beyond this point, the energy loss of the beam to the wakefield continues to increase, while the number of electrons in the ion column saturates, and the total positron yield decreases. When these two effects are quantitatively taken into account, the agreement between the measured and the calculated yield is good.

In Figure 8(b), we show the integrated positron yield in the energy range of 27-30 $\mathrm{MeV}$ (near the peak of the positron spectrum at this higher density) versus the CTR signal for three different Li vapor lengths. Higher values of CTR energy correspond to a higher loss of beam energy to the wake. Therefore the positron yield is expected to decrease as CTR energy increases for a given plasma length. When the wakefield loss is relatively small, for example for the CTR bin of 125, the positron yield increase from 13 $\mathrm{cm}$ to $22.5 \mathrm{~cm}$ and from $22.5 \mathrm{~cm}$ to $30.5 \mathrm{~cm}$ is roughly in proportion to the increase in the plasma length. However, when the average energy loss to the wake is large, for example for the CTR bin of 375, there is no further yield increase if the length is increased from 22.5 to $30.5 \mathrm{~cm}$ and only a small change from $13 \mathrm{~cm}$ to $22.5 \mathrm{~cm}$. This is due to higher beam-plasma coupling as the density increases since the energy loss dominates at high $n_{p e}$.

The agreement between the experiment and calculation provides confidence that this model can be extended to design a positron source with more optimum parameters. A schematic of such a source is shown in figure 9. The source uses a 1-m Cs plasma with a $0.5 X_{o} \mathrm{~W}$ target that resides 2-m downstream from the exit of the plasma. This distance provides space for a dipole magnet to be installed that would deflect the electrons away from the target, eliminating critical thermal stress issues. We propose to use Cs because of its lower ionization potential compared to $\mathrm{Li}$ in order to maximize the number of beam electrons that reside in the ion column. 

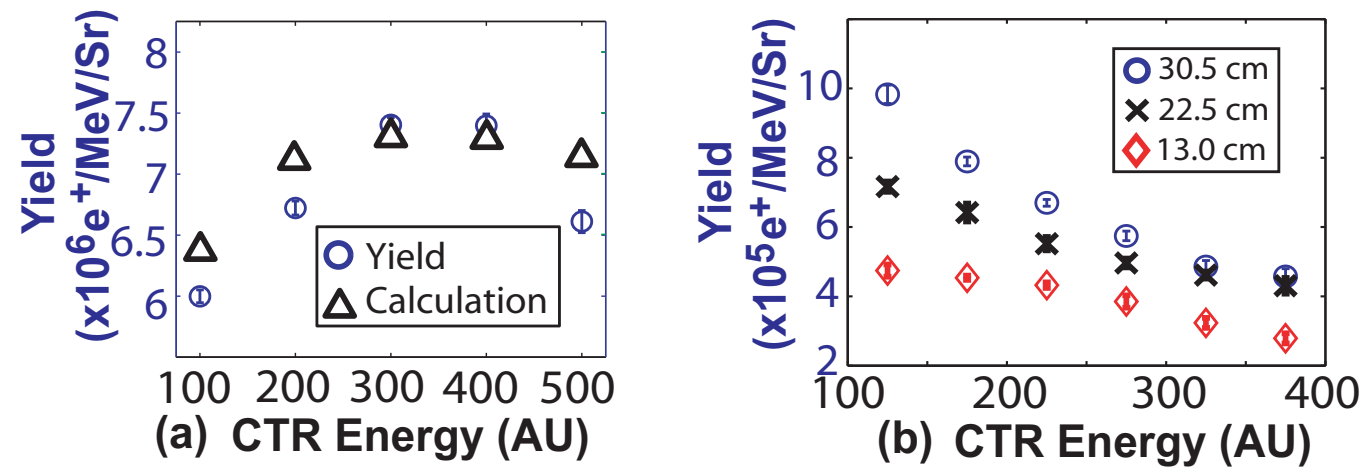

FIGURE 8. (a) The integrated positron yield in the 4-20 MeV energy range versus CTR signal. Also plotted is the calculated positron yield for the same energy range within the $11 \mathrm{~cm}$ plasma with $n_{p e}=1 \times 10^{17} \mathrm{~cm}^{-3}$. (b) Integrated positron yield in the $27-30 \mathrm{MeV}$ energy range versus CTR signal for 3 different plasma lengths with $n_{p e}=2.7 \times 10^{17} \mathrm{~cm}^{-3}$. The CTR scale may be different between the two plots.

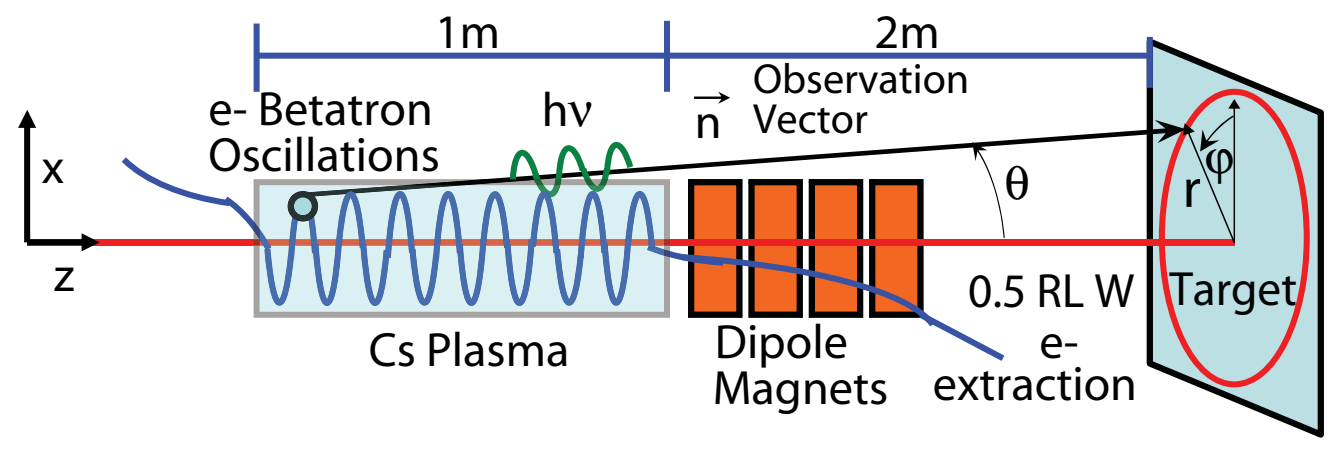

FIGURE 9. Proposed positron source experiment schematic.

The simulation input parameters are determined using QuickPIC [17]. Parameters were chosen that had already been used experimentally at SLAC. Using QuickPIC, we conclude that an "ideal" case would have a 3-D Gaussian beam of $50 \mathrm{GeV}$ electrons with $N_{b}=4 \times 10^{10}$ with $\sigma_{r}=9 \mu \mathrm{m}, \sigma_{z}=35 \mu \mathrm{m}$, and $n_{p e}=3 \times 10^{17} \mathrm{~cm}^{-3}$. This case has promise, as it collects $0.23 e^{+} /$beam $e^{-}$from $1-30 \mathrm{MeV}$ and $0.44 e^{+} /$beam $e^{-}$from 1-50 $\mathrm{MeV}$. These approach the initial goal of $1-2 e^{+} /$beam $e^{-}$. Note that this result does not include the flux concentrator used in positron systems that enhances collection efficiency [1].

\section{CONCLUSION}

In conclusion, we have investigated a new scheme for generating positrons based on betatron X-rays emitted in a plasma wiggler. The positron yield as a function of plasma density, plasma length and beam pulse length has been measured. The measured positron spectra are in excellent agreement with those expected from the calculated X-ray spectral 
yield from the plasma wiggler. Optimization of this source requires that one should

operate at as high a density as possible while still satisfying the condition $n_{p e}<n_{b}$. However, it is desirable to use as long a $\sigma_{z}$ and as large a $\sigma_{x, y}$ as possible to maximize the radiation power relative to the wake energy loss.

\section{ACKNOWLEDGMENTS}

This work was funded with DOE grants: DE-FG02-92ER40727, DE-FG03-92ER40745, DE-AC02-76SF00515 (SLAC), and NSF grant: No. PHY-0406758.

\section{REFERENCES}

1. SLC Design Handbook, Ch. 5, pp.15-79, R. Erickson, ed. (1984).

2. K. Flottmann, Conversion of Undulator Radiation, edited by Chao and Tigner in Handbook of Accelerator Physics and Engineering, 433 (1998).

3. G. Alexander et al., Undulator Based Production of Polarized Positrons, SLAC-TN-04-018, 2003.

4. J. Frisch, 1997 Proceedings of the Workshop on New Kinds of Positron Sources for Linear Colliders, Stanford Linear Accelerator Center, CA (1997)

5. T. Takahashi, 1997 Proceedings of the Workshop on New Kinds of Positron Sources for Linear Colliders, Stanford Linear Accelerator Center, CA (1997)

6. S. Wang et al., Phys. Rev. Lett. 88, 135004 (2002).

7. C.E. Clayton et al., Phys. Rev. Lett. 88, 154801 (2002).

8. E. Esarey et al., Phys. Rev. E 65, 056505 (2002).

9. I. Kostyukov et al., Phys. Plasmas 10, 4818 (2003).

10. W. Nelson, H. Hirayama and D. Rogers, SLAC-R-265, 1985.

11. M.J. Hogan, Phys. Rev. Lett. 95, 054802 (2005).

12. D.L. Bruhwiler et al., Phys. Plasmas, 102022 (2003).

13. C.O'Connell et al., to be submitted to PR-STAB, 2006.

14. C. Joshi et al., Phys. Plasmas, 9 , 1845 (2002).

15. C.D. Barnes, Ph.D. Thesis, Stanford University, 2006.

16. K.A. Marsh et al., Proceedings of the 2005 Particle Accelerator Conference (2005).

17. C. Huang et al., Proceedings of the 2003 Particle Accelerator Conference (2003).

18. D. K. Johnson, Ph.D Thesis, UCLA, 2006.

19. D.H. Whittum and A. Sessler, Phys. Rev. Lett. 64, 2511 (1990). 